\title{
Processos de enfrentamento do estresse e sintomas depressivos em pacientes portadores de retocolite ulcerativa idiopática
}

\author{
STRESS COPING STRATEGIES AND DEPRESSIVE SYMPTOMS \\ AMONG ULCERATIVE COLITIS PATIENTS
}

\section{PROCESOS DE ENFRENTAMIENTO DEL ESTRÉS Y SÍNTOMAS DEPRESIVOS EN PACIENTES PORTADORES DE RECTOCOLITIS ULCEROSA IDIOPÁTICA}

\section{Ana Lúcia Siqueira Costa', Eliane Corrêa Chaves ${ }^{2}$}

\section{RESUMO}

Neste estudo se investigou as formas de enfrentamento do estresse e a presença dos sintomas depressivos em 100 indivíduos portadores de retocolite ulcerativa idiopática (RCUI), usuários do Ambulatório de Doenças Inflamatórias do HCFMUSP, que foram comparados com 100 indivíduos acompanhantes de pacientes de outra unidade da mesma instituição, isentos da doença. A metodologia consistiu de aplicar aos dois grupos o Inventário de Estratégias de Enfrentamento de Folkman e Lazarus e o Inventário de Depressão de Beck. As estratégias de enfrentamento mais utilizadas pelo grupo de doentes foram Suporte Social (47\%) e Reavaliação Positiva (40\%), e no grupo controle ocorreu predomínio da estratégia Reavaliação Positiva (52\%). Quanto ao transtorno de humor, $71 \%$ dos portadores de RCUI não apresentavam sintomas depressivos (contra 78\% do grupo controle), 9\% apresentavam disforia (contra $9 \%$ do grupo controle) e $20 \%$ sintomas depressivos (contra $13 \%$ do grupo controle).

\section{DESCRITORES}

Estresse.

Depressão.

Colite ulcerativa/psicologia.

Psicometria/métodos.

\section{ABSTRACT}

This study investigated associations between the ways for coping with stress and the presence of depressive symptoms in a sample of 100 ulcerative colitis (UC) outpatients who are treated at the Inflammatory Bowel Disease Clinic at the School of Medicine of the University of São Paulo's Hospital das Clínicas, in the city of São Paulo. Patients were compared to a control group made up of 100 healthy subjects who kept company to other patients at another unit of the same hospital. To both groups were applied the Folkman and Lazarus's Coping Strategies Questionnaire and the Beck Depression Inventory. Coping strategies most referred to by patients were social support (47\%) and positive reappraisal (40\%); in the control group, the most reported coping strategy was positive reappraisal $(52 \%)$. As to humor disorders, $71 \%$ of the UC patients were found to show no depressive symptoms (control group's: 78), 9\% presented dysphoria (control group: 9\%), and $20 \%$ showed depressive symptoms (control group: 13\%).

\section{KEY WORDS}

Stress.

Depression.

Ulcerative colitis/psycology.

Psychometric/methods.

\section{RESUMEN}

En este estudio se investigó las formas de enfrentamiento del estrés y la presencia de los síntomas depresivos en 100 individuos portadores de rectocolitis ulcerosa idiopática (RCUI), usuarios del Consultorio Externo de Enfermedades Inflamatorias del HCFMUSP, que fueron comparados con 100 individuos acompañantes de pacientes de otra unidad de la misma institución, libres de la enfermedad. A metodología consistió en aplicar a los dos grupos el Inventario de Estrategias de Enfrentamiento de Folkman y Lazarus y el Inventario de Depresión de Beck. Las estrategias de enfrentamiento más utilizadas por el grupo de enfermos fueron Soporte Social (47\%) y Reevaluación Positiva (40\%), y en el grupo control predominó la estrategia Reevaluación Positiva (52\%).En cuanto al trastorno del humor, $71 \%$ de los portadores de RCUI no presentaron síntomas depresivos (contra el 78\% del grupo control), 9\% presentaron disforia (contra el $9 \%$ del grupo control) y el $20 \%$ síntomas depresivos (contra el $13 \%$ del grupo control).

\section{DESCRIPTORES}

Estrés.

Depresión.

Colitis ulcerosa/psicologia.

Psicometria/metodos.
* Extraído da tese "Pro cessos de enfrentamento do estresse e sintomas depressivos em pacientes portadores de retocolite ulcerativa idiopática", Escola de Enfermagem da Universidade de São Paulo (EEUSP), 2003.

1 Enfermeira. Doutora em Enfermagem pela EEUSP ana.calache@uol.com.br 2 Enfermeira. Professora Doutora do Departamento de Enfermagem MédicoCirúrgica da EEUSP. 


\section{INTRODUÇÃO}

Nos últimos anos muito se tem falado sobre a relação existente entre as doenças e os aspectos mentais. Ao longo da história o enfoque dado para a relação, os processos emocionais e os somáticos também estiveram presentes, mas foram se modificando conforme o momento religioso e o avanço técnico-científico pelo homem atingido ${ }^{(1)}$. Na esfera psicanalítica, diferentes abordagens para esta relação foram surgindo, sendo a linha Freudiana o principal referencial teórico. Nesta abordagem, observa-se o desenvolvimento de métodos terapêuticos, ressaltando a importância dos fatores psíquicos na dinâmica de toda doença. Também a Escola Psicossomática de Chicago criou a concepção do organismo como uma estrutura integrada, com todas as suas partes inter-relacionadas formando uma única unidade, sendo que a dicotomia psique-soma não se justifica, e assim, os fenômenos emocionais ocorrem em uma cadeia de eventos fisiológicos, através do Sistema Nervoso Central ${ }^{(2)}$.

Seguindo este enfoque, os primeiros estudos na era moderna que relacionam a influencia dos fatores externos e a estabilidade orgânica, foram desenvolvidos onde as doenças humanas foram estudadas em uma perspectiva de interação entre o corpo, a mente e o ambiente $^{(3)}$.

Desenvolveu-se o conceito de estresse nas ciências biológicas com destaque para as manifestações neuroendócrinas que ocorrem no indivíduo frente aos estímulos internos ou externos, sendo que para este autor, as doenças surgem como uma falha no sistema de controle homeostático orgânico, ou seja, ocorre uma incapacidade do organismo para elaborar suas próprias defesas frente à persistência de um agente estressor ${ }^{(4)}$.

No entanto, apesar de as inúmeras perspectivas de análise com o intuito de melhor definir esta relação, algumas lacunas ainda se fazem presentes quando se quer abordar o indivíduo doente e não somente a doença do indivíduo. Assim, o desenvolvimento deste estudo permitiu conhecer algumas facetas da relação doença e os aspectos emocionais, fato este que irá permitir maior compreensão do doente dentro do seu contexto de vida e ainda possibilitar uma melhoria na qualidade da assistência prestada.

A perspectiva teórica desta pesquisa baseia-se na concepção da doença que salienta sua interação com os aspectos emocionais, vivenciados pelos portadores de RCUI sob a luz de uma abordagem objetiva.

Assim, optou-se em analisar as características clínicas da doença nos portadores de RCUI em associação com o modo de enfrentamento destes indivíduos às situações de estresse e a possibilidade dos sintomas depressivos estarem envolvidos nesta relação.

Embora o enfoque norteador para análise desta pesquisa não contemple as emoções como fator único e nem causal na etiologia de RCUI, admite-se a multifatoriedade, onde as funções de natureza emocionais, fisiológicos, fisiopa-tológicas se interagem construindo implicações múltiplas e complexas que podem ser percebidas clinicamente por meio de distúrbios de ordem física, mental e comportamental.

A escolha de indivíduos portadores de RCUI como sujeitos desta pesquisa foram várias. $\mathrm{O}$ conhecimento sobre a doença e a percepção de sofrimento vivenciado pelos mesmos foi uma das razões, mas, sobretudo é pertinente o estudo, pelo aumento considerável de doentes nos últimos anos, a gravidade dos sintomas e suas conseqüências no seu contexto de vida, a indeterminação do aspecto evolutivo da doença e suas conseqüências de insegurança e medo que imprime na vida futura, a cronicidade da doença e o uso constante de medicação, e principalmente, o fator psicogênico aí envolvido, admitindo-se que os conflitos emocionais podem influir na evolução da doença ou na precipitação da recidiva, o que é comumente observado e relatado pelos próprios pacientes.

Embora diversos fatores sejam apontados como responsáveis pela etiologia da RCUI, a interação entre a susceptibilidade genética, ambiental e imunológica parece ser o mais aceito.

Assim estudar os processos de enfrentamento das situações de estresse nestes doentes foi pertinente na medida em que se observa a existência de uma inter-relação entre os sistemas neuroendócrino e imunológico e a influência dos fatores de estresse no desequilíbrio homeostático. Sabe-se que os indivíduos expostos a situações de estresse por longos períodos estão mais sujeitos às doenças ou ao agravamento daquelas já existentes como consequiência de um excesso de desgaste orgânico ou como um excesso de reações físicas em decorrência de situações de tensão.

Da mesma forma, analisar a presença dos sintomas depressivos nestes doentes se deu em função das pesquisas que apontam a existência de uma comunicação bidirecional entre o sistema imunológico e o neuroendócrino, o que caracteriza a influência dos fatores psíquicos no surgimento das alterações imunológicas, como as que ocorrem na doença de $\mathrm{RCUI}^{(5-7)}$. E, pode-se dizer também, que a depressão se constitui em um dos sintomas dos processos de estresse. 
Portanto, esta pesquisa teve como objetivo verificar, em indivíduos portadores de RCUI, qual é a associação existente entre as características clínicas da doença, os processos de enfrentamento do estresse e os sintomas depressivos. No entanto, para verificar as peculiaridades (singularidade, especificidades) deste grupo de indivíduos quanto aos processos de enfrentamento e os sintomas depressivos foi necessário: identificar e comparar o mais freqüente evento estressor entre o grupo de portadores de RCUI e o grupo controle; identificar e comparar o mais freqüente fator de enfrentamento entre o grupo de doentes e o grupo controle; identificar e comparar a mais freqüente categoria de sintomas depressivos entre o grupo dos portadores de RCUI e o grupo controle e caracterizar as manifestações clínicas da RCUI no grupo de indivíduos doentes.

\section{METODOLOGIA}

A construção metodológica desta pesquisa foi do tipo transversal de correlação, exploratório descritivo e se constitui de uma análise comparativa entre um grupo de indivíduos isentos da doença, sendo as variáveis processos de enfrentamento do estresse e sintomas depressivos, os principais aspectos desta abordagem.

Como local de estudo foi escolhido o Ambulatório de Doenças Inflamatórias do Cólon, mantido pelo Serviço de Coloproctologia do Departamento de Cirurgia da Faculdade de Medicina da Universidade de São Paulo e para a formação do grupo controle foi escolhido o Serviço de Endoscopia Digestiva e Broncoscopia ambos localizados no Prédio dos Ambulatórios que pertence ao Hospital das Clínicas da Faculdade de Medicina da Universidade de São Paulo (HCFMUSP). A escolha deste Serviço se deu pelo grande volume de indivíduos para realização de exame, fato este que facilitaria a coleta de dados.

Para a inclusão dos indivíduos doentes neste estudo foram estabelecidos os seguintes critérios:

- ser portadores de RCUI em qualquer fase de evolução da doença seja ela crônica, recidivante ou de acalmia;

- ter idade superior a 21 anos;

- aceitar participar da pesquisa e proceder à concordância por escrito no documento Termo de Consentimento Livre e Esclarecido do HCFMUSP;

- não fazer uso de drogas antidepressivas ou ansiolíticas, no período mínimo de um mês antecedente à participação da pesquisa;

Para a inclusão do grupo controle os critérios estabelecidos foram:

- não ser portador de RCUI;

- ter idade superior a 21 anos;
- aceitar participar da pesquisa e proceder à concordância por escrito no documento Termo de Consentimento Livre para Participação da Pesquisa desenvolvido para utilização da pesquisa;

- não fazer uso de drogas antidepressivas ou ansiolíticas, no período mínimo de um mês antecedente à participação da pesquisa;

- não ser portador de qualquer tipo de doença que fosse de seu conhecimento;

- ser do sexo masculino ou feminino de modo a equiparar-se numericamente à distribuição por sexo do grupo de pacientes.

Após aprovação do projeto de pesquisa expedida pela Comissão de Ética do Departamento de Gastroenterologia da Faculdade de Medicina da Universidade de São Paulo e pela Comissão de Ética para Análise de Projetos de Pesquisa-CAPPesq da Diretoria Clínica do HCFMUSP, a coleta de dados iniciou-se em fevereiro e estendeu-se a maio de 2002 e foi realizada pela própria pesquisadora. $\mathrm{O}$ encerramento da coleta baseou-se no critério quantitativo e não temporal, quando foram alcançados os 100 indivíduos em cada grupo. A escolha por este número de indivíduos se deu por acreditar que seria suficiente para que os testes estatísticos fossem realizados com a fidedignidade desejável dos resultados.

Tendo em vista conhecer as diversas características sociodemográficas, os processos de enfrentamento e os sintomas depressivos nos dois grupos de estudos e, ainda as características da doença nos portadores de RCUI, foram utilizados:

- o instrumento de coleta de dados sóciodemográficos, contendo informações que pudessem caracterizar os indivíduos participantes desta pesquisa e consistiu dos seguintes itens: sexo, idade, cor, escolaridade, situação conjugal, religião e prática religiosa, pessoas com quem compartilha a moradia, ocupação e cidade em que reside.

- o instrumento de coleta de dados sobre a patologia de RCUI, o qual irá fornecer informações sobre as condições clínicas dos portadores de RCUI e abrange as mais importantes manifestações necessárias para caracterizar a gravidade da doença e de maior relevância para a análise pretendida. Os itens aí incluídos referem-se aos sintomas digestivos, à extensão da doença no cólon, à necessidade de cirurgia para tratamento da doença, à presença de manifestações extra-intestinais, ao uso de medicamentos e o tempo de diagnóstico da doença.

- o Inventário de Estratégias de Coping (Enfrentamento) de Folkman e Lazarus (1980) que se compõe de 46 itens para identificar 46 estratégias de enfrentamento de situações de estresse, agrupadas em oito diferentes fatores: Confronto, Afastamento, Autocontrole, Suporte social, Aceitação da 
responsabilidade, Fuga esquiva, Resolução do problema e Reavaliação positiva ${ }^{(8-9)}$.

- o Inventário de Avaliação de Depressão de Beck foi construído com 21 categorias de sintomas e atitudes características das manifestações de depressão e envolve manifestações de humor, vegetativas, sociais, cognitivas e de irritabilidade. As categorias foram agrupadas em ausência de sintomas, presença de disforia e de sintomas indicativos de depressão ${ }^{(10-11)}$.

Antes de iniciar a coleta de dados foi realizado um préteste com seis pacientes com a finalidade de avaliar se as orientações para o preenchimento do instrumento eram adequadas, se os termos utilizados eram compreensíveis e para avaliar o tempo dispensado para o preenchimento do instrumento. Devido à baixa escolaridade da população do estudo, ao grande número de itens em cada instrumento e a dificuldade para proceder à leitura do instrumento, houve necessidade de adequação de alguns termos e a leitura dos inventários passou a ser realizada pela própria pesquisadora.

Para a análise dos dados foi realizado inicialmente, o agrupamento dos dados sóciodemográficos, as estratégias de enfrentamento, os sintomas depressivos e as características clínicas da doença para a seguir proceder a análise estatística.

Para avaliar a adequação e a qualidade dos instrumentos propostos foi utilizado o teste de confiabilidade alfa de Cronbach para o Inventário de Estratégias de Enfrentamento para o total dos 200 indivíduos que compõe a população do estudo, para o grupo de doentes e para o grupo controle em separados. O mesmo procedimento foi utilizado para cada fator, da mesma forma o alfa de Cronbach foi utilizado para o Inventário de Depressão de Beck ${ }^{(12)}$.

Para comparar a pontuação máxima encontrada em cada categoria de sintomas depressivos foi utilizado o teste de postos para dados não-emparelhados de Kruskal-Wallis ${ }^{(13)}$.

Para se comparar estatisticamente a distribuição das variáveis entre o grupo de indivíduos com RCUI e o grupo controle, para se conhecer a associação entre os fatores de enfrentamento mais utilizados e a presença ou ausência de sintomas depressivos nos dois grupos de estudo e ainda para se conhecer a associação existente entre os fatores de enfrentamento mais utilizados, a presença ou ausência de sintomas depressivos e as características clínicas da doença nos portadores de RCUI foi utilizado o teste de qui-quadrado.

Em todos os testes foi utilizado o nível de significância de $5 \%$.

\section{RESULTADOS E DISCUSSÃO}

Inicialmente, verificou-se a confiabilidade do Inventário de Estratégias de Enfrentamento de Folkman e Lazarus para esta pesquisa, uma vez que os valores do alfa de Cronbach foram superiores ao valor mínimo aceitável, tanto para os dois grupos de indivíduos em separado, como também, para o total da população em conjunto.

Quanto aos fatores de enfrentamento pode-se observar que os fatores Confronto, Suporte social, Aceitação de responsabilidade e Reavaliação positiva apresentam valores de alfa de Cronbach superiores a 0,05 e são comuns entre os dois grupos do estudo, sendo, portanto, estes fatores escolhidos para a análise estatística (Tabelas 1 e 2).

Tabela 1 - Resultados dos coeficientes da correlação alfa de Cronbach para o Inventário de Estratégias de Enfrentamento de Folkman e Lazarus, segundo os fatores e o número de itens para o grupo de indivíduos doentes. São Paulo - 2002

\begin{tabular}{lcc}
\hline Fatores de enfrentamento & $\begin{array}{c}\text { Número } \\
\text { de Itens }\end{array}$ & Alfa \\
\hline Confronto & 6 & $\mathbf{0 , 5 3}$ \\
Afastamento & 7 & 0,42 \\
Autocontrole & 5 & 0,33 \\
Suporte social & 6 & $\mathbf{0 , 7 2}$ \\
Aceitação da responsabilidade & 7 & $\mathbf{0 , 5 9}$ \\
Fuga esquiva & 2 & $\mathbf{0 , 5 4}$ \\
Resolução do problema & 4 & $\mathbf{0 , 6 6}$ \\
Reavaliação positiva & 9 & $\mathbf{0 , 6 6}$ \\
\hline
\end{tabular}

Tabela 2 - Resultados dos coeficientes da correlação alfa de Cronbach para o Inventário de Estratégias de Enfrentamento de Folkman e Lazarus, segundo os fatores e o número de itens para o grupo controle. São Paulo - 2002

\begin{tabular}{lcc}
\hline Fatores de enfrentamento & $\begin{array}{c}\text { Número } \\
\text { de Itens }\end{array}$ & Alfa \\
\hline Confronto & 6 & $\mathbf{0 , 5 1}$ \\
Afastamento & 7 & 0,46 \\
Autocontrole & 5 & 0,34 \\
Suporte social & 6 & $\mathbf{0 , 5 5}$ \\
Aceitação da responsabilidade & 7 & $\mathbf{0 , 6 1}$ \\
Fuga esquiva & 2 & 0,40 \\
Resolução do problema & 4 & 0,32 \\
Reavaliação positiva & 9 & $\mathbf{0 , 6 7}$ \\
\hline
\end{tabular}

Com relação ao Inventário de Depressão de Beck verifica-se que em todas as situações, os valores de alfa de Cronbach foram superiores a 0,05 o que garante a boa confiabilidade do instrumento da pesquisa. 
Quanto às características sóciodemográficas da população do estudo pode-se constatar semelhança entre os dois grupos, fato este que favoreceu a adequação metodológica desta pesquisa tendo em vista os objetivos propostos. Ainda com relação aos dados sóciodemográficos observa-se predominância de indivíduos do sexo feminino, numa faixa etária entre 21 e 40 anos, de cor branca. A maioria vive com companheiro, tem como escolaridade o curso médio completo ou incompleto, são católicos e praticam sua crença religiosa. Os indivíduos moram, a grande maioria, com os seus familiares, possuem como ocupação vínculo empregatício e residem predominantemente na capital de São Paulo. Com relação aos resultados aqui encontrados sobre a idade e cor, existe concordância com os resultados encontrados em outros estudos de mesma natureza, porém, quanto ao sexo os resultados mostram-se diferentes, e isto pode refletir não especificamente alguma característica relacionada à doença, mas sim, o universo dos portadores de RCUI que procuram o Ambulatório de Doenças Inflamatórias do Cólon, não sendo o caso aqui de pesquisar as razões deste ambulatório ser mais procurado por mulheres do que por homens.

Quando os indivíduos foram solicitados para escolherem uma situação de estresse para proceder ao preenchimento do Inventário de Enfrentamento, verifica-se que a categoria dificuldades pessoais, em especial a própria doença, foi predominante entre os portadores de RCUI. Diferentemente no grupo controle, a categoria família, com ênfase na doença em família, foi o mais citado. A diferença entre ambos mostrou-se estatisticamente significativa (Tabela 3 ).
Tabela 3 - Distribuição dos 100 indivíduos com RCUI e dos 100 indivíduos do grupo controle, segundo as categorias dos eventos vitais. São Paulo - 2002

\begin{tabular}{lcc}
\hline $\begin{array}{l}\text { Categorias de } \\
\text { eventos vitais }\end{array}$ & RCUI(\%) & Controle (\%) \\
\hline Trabalho & 8 & 16 \\
Perda do suporte social & 8 & 14 \\
Família & 18 & 44 \\
Dificuldades pessoais & 60 & 17 \\
Finanças & 6 & 9 \\
\hline Total & 100 & 100 \\
\hline$\dot{\div}^{2}=39,8192$ & & \\
$\mathrm{p}=0,000$ & &
\end{tabular}

A situação de estresse citada pelos portadores de RCUI justifica-se pelas próprias características da doença e a forma de apresentação dos sintomas que, dependendo da intensidade, são debilitantes e representam uma ameaça iminente quanto à vida futura ${ }^{(14-16)}$.

Ao analisar os processos de enfrentamento mais utilizados verificou-se que o fator Suporte social foi utilizado pelo maior número de doentes, seguido do fator Reavaliação positiva. Diferentemente, o fator Reavaliação positiva foi predominante no grupo controle. E esta diferença entre os resultados mostrou-se estatisticamente significativa (Tabela 4).

Tabela 4 - Distribuição dos 100 indivíduos com RCUI e dos 100 indivíduos do grupo controle, segundo os mais freqüentes fatores de enfrentamento. São Paulo - 2002

\begin{tabular}{lccc}
\hline Fatores de enfrentamento & RCUI(\%) & Controle(\%) & Total \\
\hline Aceitação da responsabilidade ,Reavaliação positiva & & & \\
e Suporte social em conjunto & 2 & 8 & 10 \\
Aceitação da responsabilidade & 11 & 8 & 92 \\
Reavaliação positiva & 40 & 32 & 79 \\
Suporte social & 47 & 100 & 200 \\
\hline Total & 100 & &
\end{tabular}

O suporte social é um fator psicossocial positivo que pode ajudar o indivíduo a melhorar o efeito indesejado do estresse e a sua resposta à doença de RCUI. O isolamento social pode atrapalhar a habilidade do indivíduo para enfrentar sua condição de doença, e assim favorecer a coexistência de alterações psiquiátricas ao longo da condição clínica da doença ${ }^{(17)}$. Vale ressaltar que o Suporte social para os doentes desta pesquisa se refere predominantemente aos familiares e à religião e, não pode esquecer que a religiosidade foi declarada em $60,4 \%$ dos doentes desta pesquisa. Diferentemente, outros pesquisadores ${ }^{(18)}$ relatam que os indiví- duos portadores de RCUI utilizam predominantemente o fator Resolução de problema e acrescentam que estes doentes parecem estar mais bem adaptados às suas experiências com a doença.

No que se refere aos sintomas depressivos, verifica-se que a diferença da pontuação total obtida entre os dois grupos do estudo não foi estatisticamente significativa. Os resultados das pontuações obtidas no Inventário de Depressão de Beck mostram que a maioria dos indivíduos foi classificada na categoria de ausência de sintomas depressivos (Tabela 5). 
Tabela 5 - Distribuição dos 100 indivíduos com RCUI e dos 100 indivíduos do grupo controle, segundo a ausência de sintomas depressivos, presença de disforia ou de sintomas indicativos de depressão. São Paulo - 2002

\begin{tabular}{lcc}
\hline Sintomas de depressão & RCUI(\%) & Controle (\%) \\
\hline Ausente & 71 & 78 \\
Disforia & 9 & 9 \\
Presente & 20 & 13 \\
\hline Total & 100 & 100 \\
\hline$\dot{\div}^{2}=1,8137$ & & \\
p $=0,404$ & &
\end{tabular}

Pode-se constatar que a maioria dos doentes que apresenta ausência de sintomas depressivos utiliza predominantemente as estratégias de enfrentamento contidas no fator Suporte social (Tabela 6). Diferentemente nos indivíduos do grupo controle (Tabela 7), observa-se que aqueles com ausência de sintomas depressivos utilizam predominantemente o fator Reavaliação positiva, sendo que a comparação dos resultados entre as diversas categorias mostrou-se com significância estatística.

Tabela 6 - Distribuição dos 100 indivíduos com RCUI, segundo os fatores de enfrentamento mais freqüentes e ausência de sintomas depressivos, presença de disforia ou de sintomas indicativos de depressão. São Paulo - 2002

\begin{tabular}{|c|c|c|c|c|}
\hline \multirow[t]{2}{*}{ Fatores de enfrentamento } & \multicolumn{4}{|c|}{ Sintomas de depressão } \\
\hline & $\begin{array}{l}\text { Ausente } \\
\mathrm{n}(\%)\end{array}$ & $\begin{array}{l}\text { Disforia } \\
\text { n(\%) }\end{array}$ & $\begin{array}{l}\text { Presente } \\
\text { n(\%) }\end{array}$ & $\begin{array}{l}\text { Total } \\
\mathrm{n}(\%)\end{array}$ \\
\hline $\begin{array}{l}\text { Aceitação da responsabilidade, } \\
\text { Reavaliação positiva e Suporte }\end{array}$ & & & & \\
\hline social em conjunto & $2(2,81)$ & $0(0)$ & $0(0)$ & 2 \\
\hline Reavaliação positiva & $29(40,84)$ & $3(33,34)$ & $8(40,00)$ & 40 \\
\hline Suporte social & $34(47,90)$ & $4(44,44)$ & $9(45,00)$ & 47 \\
\hline Total n(\%) & $71(100)$ & $9(100)$ & $20(100)$ & 100 \\
\hline
\end{tabular}

$\dot{\div}^{2}=2,7118$

$\mathrm{p}=0,844$

Tabela 7 - Distribuição dos 100 indivíduos do grupo controle, segundo os fatores de enfrentamento mais usualmente utilizados e ausência de sintomas depressivos, presença de disforia e de sintomas indicativos de depressão. São Paulo - 2002

\begin{tabular}{|c|c|c|c|c|}
\hline \multirow[t]{2}{*}{ Fatores de enfrentamento } & \multicolumn{4}{|c|}{ Sintomas de depressão } \\
\hline & $\begin{array}{c}\text { Ausente } \\
\mathrm{n}(\%)\end{array}$ & $\begin{array}{l}\text { Disforia } \\
\mathrm{n}(\%)\end{array}$ & $\begin{array}{c}\text { Presente } \\
\text { n(\%) }\end{array}$ & $\begin{array}{l}\text { Total } \\
\mathrm{n}(\%)\end{array}$ \\
\hline Aceitação da responsabilidade, & & & & \\
\hline $\begin{array}{l}\text { social em conjunto } \\
\text { Aceitação da responsabilidade }\end{array}$ & $\begin{array}{l}7(8,97) \\
3(3,84)\end{array}$ & $\begin{array}{l}0(0) \\
0(0)\end{array}$ & $\begin{array}{l}1(7,69) \\
5(38,46)\end{array}$ & $\begin{array}{l}8 \\
8\end{array}$ \\
\hline Reavaliação positiva & $41(52,57)$ & $7(77,78)$ & $4(30,77)$ & 52 \\
\hline Suporte social & $27(34,62)$ & $2(22,22)$ & $3(23,08)$ & 32 \\
\hline Total n(\%) & $78(100)$ & $9(100)$ & $13(100)$ & 100 \\
\hline
\end{tabular}

$\div^{2}=21,3357$

$\mathrm{p}=0,002$

A rede de apoio parece suprir uma função no preenchimento e manutenção dos afetos, evitando, assim, os sintomas depressivos $^{(19)}$. A utilização dessas estratégias, por alguns indivíduos, denota limitação na esfera cognitiva e na mobilização de recursos para o enfrentamento das situações. Dessa forma, verifica-se uma maior predisposição para a associação dos sintomas depressivos, as alterações somáticas e o referido fator ${ }^{(20-21)}$. 
Apesar de a categoria ausência de sintomas depressivos ser predominante nos grupos estudados, o transtorno do humor está presente em $29 \%$ dos doentes e em $22 \%$ do grupo controle. E no que tange aos sintomas depressivos, os portadores de RCUI os apresentam em maior porcentagem quando comparados ao grupo controle.

Mesmo considerando que a diferença de pontuação entre os dois grupos analisados não apresentou significância estatística, os percentuais encontrados mostram resultados relevantes. Quando comparado este resultado com o da população geral, observa-se que a prevalência destes sintomas varia de 15 a $20 \%{ }^{(22)}$. Em estudo ${ }^{(23)}$ mostrou-se que $15,8 \%$ da população do Rio de Janeiro que procura os serviços primários de assistência apresentam sintomas depressivos.

Vários aspectos podem ser citados como contribuintes para o fato de os doentes estudados nesta pesquisa apresentarem tendência a sintomas depressivos. Em relação ao sexo, verifica-se que os sintomas depressivos são mais prevalentes em mulheres do que homens, o que é consistente com a maior proporção de mulheres entre os doentes aqui estudados ${ }^{(24)}$. Outro aspecto observado é a dificuldade de adaptação desses indivíduos frente à situação de doença. Fato este percebido pela tentativa dos mesmos deslocarem o lócus de adaptação para o fator Suporte social como fonte de apoio. Ainda outro aspecto é a expressão de tristeza e choro observada muitas vezes pela pesquisadora no momento de preenchimento dos inventários da pesquisa, e, por último, vale ressaltar que os doentes estudados apresentaram pontuação total mais elevada em 14 sintomas quando comparado com o grupo controle, principalmente os sintomas cognitivo-afetivos, e aqueles que expressam sentimentos de tristeza, pessimismo, culpa, indecisão e mesmo idéia suicida, sendo estes mais indicativos de suspeita de depressão.

Ao analisar especificamente os indivíduos com maior pontuação dos sintomas depressivos, verifica-se que estes estão em maioria, conforme a presença dos sintomas digestivos da doença como diarréia, dor abdominal, hemorragia digestiva baixa, vômito, náuseas, anorexia e tenesmo. $\mathrm{O}$ mesmo se verifica entre os indivíduos que apresentam o comprometimento universal da doença no cólon.

Entre os indivíduos que não realizaram o tratamento cirúrgico, não significa necessariamente, que tenham menor gravidade da doença, mas pode significar indivíduos que tenham gravidade da doença e que aguardam na fila de espera o momento para ser realizado o procedimento cirúrgico. $\mathrm{O}$ mesmo ocorre nos indivíduos com manifestações extra-intestinais, verifica-se que aqueles com sintomas depressivos estão em maior número quando apresentam estas manifestações, o que caracteriza a maior gravidade da doença.
Aqueles com sintomas depressivos também estão em maior número quando fazem uso de medicamentos. Vale ressaltar que a necessidade de medicamento denota maior gravidade da doença, assim como, os sintomas depressivos podem surgir como conseqüência de efeitos colaterais dos próprios medicamentos.

Verifica-se também que aqueles indivíduos com presença dos sintomas depressivos estão incluídos naqueles com menor tempo de diagnóstico da doença. Confirmando os achados desta pesquisa, encontraram em pesquisa que os episódios mais intensos de ansiedade e depressão foram detectados naqueles indivíduos que vivenciavam a RCUI nos primeiros anos após o diagnóstico da doença. A fase de início de tratamento se caracteriza pelo desconhecimento da própria doença e suas consequiências na vida diária. É possível que nesta incerteza estejam ainda em fase de aprendizagem tanto das características da doença, quanto das formas de lidar com elas, gerando estresse, que por sua vez acarreta ansiedade e sintomas indicativos de depressão ${ }^{(25)}$.

\section{CONCLUSÃO}

A verificação da associação entre as características clínicas da RCUI e os sintomas depressivos mostrou que esses sintomas estão mais presentes nos indivíduos em situação de maior gravidade da doença. Quanto à associação entre as características clínicas e os processos de enfrentamento mais utilizados pelos doentes, verificou-se predominância do fator Suporte social, independentemente das manifestações clínicas da doença e da presença ou ausência de sintomas depressivos. Ao identificar e comparar o mais freqüente evento estressor entre o grupo de indivíduos portadores de RCUI e o grupo controle, verificou-se que, entre os primeiros a situação de doença foi a mais citada. No grupo controle, o evento mais citado foi doença em um membro da família. Ao identificar e comparar o mais freqüente fator de enfrentamento utilizado pelo grupo de indivíduos portadores de RCUI e pelo grupo controle, constatou-se predominância dos fatores Suporte social e Reavaliação positiva entre os doentes e Reavaliação positiva no grupo controle. Ao identificar e comparar a mais freqüente categoria de sintomas depressivos entre o grupo de indivíduos portadores de RCUI e o grupo controle, verificou-se que a maioria, em ambos os grupos, não apresenta sintomas depressivos. Espera-se que os resultados alcançados nesta pesquisa proporcionem uma melhor compreensão dos indivíduos portadores desta afecção e que possa contribuir para que outros estudos sejam realizados nesta área, carente de conhecimento teórico para subsidiar a prática assistencial. 


\section{REFERÊNCIAS}

(1) Volich RM. Psicossomática: de Hipócrates à psicanálise. São Paulo: Casa do Psicólogo; 2000.

(2) Alexander F. Medicina psicossomática: seus princípios e aplicações. Porto Alegre: Artes Médicas; 1989.

(3) Cannon WB. A sabedoria do corpo. São Paulo: Nacional; 1946.

(4) Selye H. Stress, a tensão da vida. São Paulo: IBRASA; 1959.

(5) Bauer ME, Gauer GJ, Nardi NB. Depressão maior e atividade do sistema imunológico. Rev ABP-APAL. 1993;15(3):87-94.

(6) Olff M. Stress, depression and immunity: the role of defense and coping styles. J Psychiatr Res. 1999;85(1):7-15.

(7) Okabe N, Kuroiwa A, Nagayama A. Role of psychological stress on IBD onset. Dig Dis Sci. 2000;45(1):32-3.

(8) Folkman S, Lazarus RS. An analysis of coping in a middle-aged community sample. J Health Soc Behav. 1980;21(3):219-39.

(9) Savóia MG, Santana PR, Mejias NP. Adaptação do inventário de estratégias de coping de Folkman e Lazarus para o português. Psicol USP. 1996;7(1/2):183-201.

(10) Beck AT, Ward CH, Mendelson M, Mock J, Erbaugh J. An inventory for measuring depression. Arch Gen Psychiatry. 1961;4(6):561-71.

(11) Gorenstein C, Andrade L. Inventário de Depressão de Beck: propriedades psicométricas da versão em Português. In: Gorenstein C, Andrade LHSG, Waldo A. Escalas de avaliação clínica em psiquiatria e psicofarmacologia. São Paulo: Lemos; 2000. Cap. 10, p. 89-95.

(12) Cronbach LJ. Coefficient alpha and the internal structure of tests. Psychometrika. 1951;16(3):297-334.

(13) Siegel S. Estatística não-paramétrica. São Paulo: McGrawHill do Brasil; 1975

(14) Lewis MC. Attributions and illness. J Psychosoc Nurs Ment Health Serv. 1988;26(4):14-7.

(15) Dudley-Brown S. Living with ulcerative colitis. Gastroenterol Nurs. 1995;19(2):60-4.
(16) Simrén M, Axelsson J, Gillberg R, Abrahamsson H, Svedlund $\mathrm{J}$, Bjornsson ES. Quality of life in inflammatory bowel disease in remission: the impact of IBS-Like symptoms and associated psychological factors. Am J Gastroenterol. 2002;97(2): $389-96$.

(17) Drossman DA. Psychosocial factors in ulcerative colitis and Crohn's disease. In: Kirsner JB. Inflammatory bowel disease. $5^{\text {th }}$ ed. Philadelphia: Saunders; 2000. Chap. 23, p. 342-57.

(18) Kinash RG, Fischer DG, Lukie BE, Carr TL. Coping patterns and related characteristics in patients with IBD. Rehabil Nurs. 1993;18(1):12-9.

(19) Delouya D. Depressão. São Paulo: Casa do Psicólogo; 2000.

(20) Lazarus RS, Folkman S. Stress, appraisal and coping. New York: Springer; 1984.

(21) Billings AG, Moos RH. Coping, stress, and social resources among adults with unipolar depression. J Pers Soc Psychol. 1984;46(4):877-91.

(22) Kaplan HI, Sadock BJ. Tratado de psiquiatria. $6^{\mathbf{a}}$ ed. Porto Alegre: Artes Médicas; 1999. v. 2.

(23) World Health Organization (WHO). The world health report 2: mental health-statistical annex [online]. Available from: < www.who.int/whr2001/2001main/ en/annex/ index.htm> [Access at 2002 nov. 29].

(24) Menezes PR, Nascimento AF. Epidemiologia da depressão nas diversas fases da vida. In: Lafer B, Almeida OP, Fráguas Júnior R, Miguel EC. Depressão no ciclo da vida. Porto Alegre: Artmed; 2000. Cap. 2, p. 29-36.

(25) Kurina LM, Goldacre MJ, Yeates D, Gill LE. Depression and anxiety in people with inflammatory bowel disease. J Epidemiol Community Health. 2001;55(1):716-20. 